\title{
Ein Messer, ein Feuer und viele offene Fragen
}

\section{Selina Steiger}

Medizinstudentin im ersten Jahr an der Universität Zürich

«Klick», der Schlüssel dreht sich, die Badezimmertür ist verschlossen. Allein um vier Uhr nachts, alles dunkel. In seinem Kopf ertönt das Klirren der scharfen Klinge, das Messer im Bauch - das Telefon zur Hand. Der Anruf wird von der Notrufzentrale innert Sekunden entgegengenommen. Eine tiefe Stichverletzung habe er sich zugefügt, ja, aber er wolle keine Hilfe. Die Drohung, sich anzuzünden, falls trotzdem Hilfe käme. Der Anrufer legt auf. Was nun? Wie handeln? Für langes Nachdenken und Abwägen bleibt der Notrufstelle keine Zeit. Sanitäter und bewaffnete Polizisten machen sich auf den Weg und stürmen das Haus des Anrufers. Das leise Zischen des Zündholzes, als es entfacht wird, ist kaum hörbar. Die letzte Möglichkeit für die Rettungstruppe, sich wieder zurückzuziehen. Retten oder nicht retten? Die Autonomie des Patienten wahren und ihn seiner eigenen Entscheidung überlassen oder Gutes tun und versuchen, einen Menschen vor sich selbst zu schützen? Ein Moment der Unsicherheit, dann bricht das Feuer aus.

Im ersten Jahr studiere ich Medizin, und schon kommen mir solche grossen Fragen wie Monster entgegengesprungen. Man soll sich mit ihnen beschäftigen und sie in ihrer Vielschichtigkeit erkennen. Im ersten Moment bin ich vom Fall von Herrn Novak betroffen, schockiert, in gewissem Masse erschüttert und kann ihn nicht verstehen. Wie schlecht muss es einem Menschen gehen, dass er sich Schaden und Schmerz in diesem Ausmass zufügt? Widerwillig bemerke ich, wie sich meine Bestürzung mit einer scheuen Faszination paart. Ist es genau diese Komplexität des Falles, die mich reizt, mehr über Herrn Novak zu erfahren? Vor meinem inneren Auge sehe ich das Geschehen mit all seinen Hintergründen in vielen einzelnen Schachteln verpackt, die sich ineinander verbergen und nur darauf warten, geöffnet zu werden. Mein Interesse ist geweckt und ich beginne auszupacken.

Für Frau Gerber in der Notrufzentrale sind solche Anrufe nichts Ungewöhnliches, aber trotzdem überkommt sie jedes Mal aufs Neue ein beklemmendes Gefühl. Was tut man, wenn der Anrufer seinen Suizidversuch zwar offen darlegt, jedoch droht, sich noch mehr Schaden zuzufügen, falls ein Rettungsversuch stattfinden sollte? Was ist Herrn Novaks Wille? Schon eine Stichverletzung im
Bauch führt meist zu lebensgefährlichen Komplikationen. Kämen auch noch schwere Verbrennungen hinzu, wäre das Sterberisiko massiv erhöht. Sollen diese Verbrennungen durch eine Rettungsaktion wirklich provoziert werden? Damit würde man dem Mann, falls er seine Drohung wahrmacht, noch mehr schaden und zudem seine Autonomie missachten. Für Frau Gerber ist erst die äusserste Schachtel sichtbar, erst das Bild, das Herr Novak willentlich von sich selbst entwirft. Hintergründe der Tat sind ihr nicht bekannt. Die vier ethischen Prinzipien «Wohltun», «nicht schaden», "Gerechtigkeit» und "Autonomie», an denen man sich orientieren sollte, um die ethisch richtige Entscheidung zu treffen, sind wie Stahlseile ineinander verlötet und widersprechen sich. Ich bin froh, nicht in Frau Gerbers Haut zu stecken. Sie entscheidet sich dafür, die Polizei zu informieren, welche gemeinsam mit dem Sanitätsteam ausrückt und die Wohnung von Herrn Novak stürmt. Dieser übergiesst sich mit Benzin und zündet sich an. Obwohl er sofort von der Polizei gelöscht wird, lassen sich schwerwiegende körperliche Schäden nicht verhindern. Herr Novak wird mit einer zu 65 Prozent verbrannten Hautoberfläche auf die Intensivstation gebracht, wo er die nächsten sechs Monate seines Lebens verbringen wird. Unnachgiebig sitze ich vor dem Schachtelberg und versuche mir den Durchblick zu verschaffen. Weshalb hat Herr Novak den Notruf verständigt, um sich danach, als Rettung nahte, noch mehr zu verletzen? War das Ganze ein Hilfeschrei? Ein lautes Rufen nach Rettung vor sich selbst? Gemäss seiner Krankenakte litt der 30-Jährige an schizophrener Psychose. Diese habe sich infolge der Ablösung von seiner Familie, die er wohl schweren Herzens in Kroatien zurückgelassen haben muss, verstärkt. In der Schweiz habe er geheiratet und sei Vater zweier Töchter geworden. Seine Krankheit sei aber bestehen geblieben. Es lässt sich also darüber streiten, ob Herr Novak zur Zeit der Tat überhaupt urteilsfähig und der Suizidversuch nicht eine böse Folge seiner Psychose gewesen ist.

Auf der Intensivstation herrscht bedrückte Stimmung. Der schwer verbrannte Patient macht es den Ärzten nicht einfach. Er liegt im Koma, sein Zustand ist kritisch. Es ist nicht klar, ob er überleben will und wird. Welche lebensrettenden Massnahmen sollen getroffen werden, 
welche nicht? Eine Patientenverfügung ist nicht vorhanden und der Wille des Patienten nicht ermittelbar. Auch Herr Novaks Frau wehrt jegliche Kontaktaufnahme ab. Angeblich habe sie sich von ihrem Mann getrennt, da dieser ihr und seinen Kindern gegenüber mehrmals gewalttätig geworden sei. Die Entscheidung liegt also in den Händen der Ärzte, in den Händen meines ZukunftIchs. Wie würde ich mich entscheiden? Herr Novak könnte im besten Fall noch ein langes Leben vor sich haben, doch für die Rehabilitation bräuchte es seine volle Motivation. Ausserdem wäre er auch bei Erfolg wahrscheinlich nie wieder in der Lage, für sich selber zu sorgen, und müsste in einem Pflegeheim wohnen.

Plötzlich muss ich an seine Frau und die beiden Mädchen denken, die schon mehrmals unter häuslicher Gewalt gelitten haben und die sich nun vielleicht zum ersten Mal sicher fühlen, da ihr Mann und Vater im Spital liegt. Missgunst und Abscheu mischen sich in meine Gefühlslage. Vielleicht verlässt Herr Novak im Falle einer Genesung das Spital mit einer noch grösseren Gewaltbereitschaft und als noch grössere Gefährdung für die beiden Schulmädchen. Wäre es also nicht doch besser, dem Ganzen seinen Lauf zu lassen? Herr Novak wollte sich doch sowieso das Leben nehmen. Noch im selben Augenblick fühle ich mich schuldig für diese Gedanken. Ich weiss genau, dass das private Familienleben des Patienten nicht in meinen Zuständigkeitsbereich gehört und auch nicht gehören darf. Trotzdem sind die Gedanken da und lassen sich nicht vertreiben. Aspekte der Folgenethik mischen sich hinzu. Dieser Patient würde Hunderttausende von Franken kosten, und die Sterbewahrscheinlichkeit bei einer solchen Verbrennung liegt bei über 80 Prozent! Wäre es nicht gerechter, dieses Geld in Patienten zu investieren, die unverschuldet im Spital liegen? Andererseits könnte genau ein solcher Einschnitt einen Wendepunkt im Leben von Herrn Novak bewirken, ihm zeigen, dass er etwas ändern muss. Er könnte wieder Sinn im Leben sehen und dankbar dafür sein, dass ihm heute sein Leben gerettet worden wäre. Ich führe einen inneren Konflikt und spüre den Druck der riesigen Verantwortung, die ein Arzt in so einem Moment über ein Menschenleben besitzt, wie eine schwere Last auf meinem Rücken. Kann ich sie tragen? Habe ich die Fähigkeit und die Kraft dazu? Wie froh bin ich, dass ich heute noch nicht diejenige bin, die entscheiden muss.

Gedankenversunken blättere ich durch den Stapel Papier mit den Arztberichten. Ich erfahre, dass sich die Ärzte nach Rücksprache mit Ethikern dazu entschieden haben, alle lebensnotwendigen Massnahmen einzuleiten. Täglich würden die Verbrennungen des Patienten gesäubert und die Verbände gewechselt. Andere Verletzungen seien nicht vorhanden. Ich stocke und blättere zurück. Habe ich mich verlesen? Mein Puls steigt, ich merke, dass ich ganz nah dran bin, auch Einblick in eine der letzten und grössten Schachteln $\mathrm{zu}$ erhalten. Tatsächlich steht schwarz auf weiss, dass trotz gründlicher Untersuchung nie eine Stichverletzung gefunden worden sei.

Wie ein Blitz durchzuckt mich diese Erkenntnis und ändert meine Sichtweise auf das ganze Geschehen erneut. Hat Herr Novak selbst ein ethisches Dilemma durchlebt? Er konnte sich nicht entscheiden, ob er sterben oder leben wollte. Vor meinen Augen sehe ich den einsamen und hilflosen Mann alleine im Badezimmer stehen, vor Verzweiflung nicht in der Lage, klar zu denken, und unfähig, mit der Situation umzugehen. Nun öffnen sich die Schachteln und ordnen sich in meinem Kopf. Das schlechte Gewissen, dass er seine Eltern in Kroatien zurückgelassen und seine Frau und Kinder misshandelt hat, verfolgt den jungen Mann. Hat er den Tod als seine Pflicht und als Wiedergutmachung gegenüber der Familie gesehen? Erhoffte er sich so eine Erlösung von seiner Einsamkeit und seinen Schuldgefühlen? Aber er hätte doch noch ein langes Leben vor sich haben und die Beziehung zu seinen Kindern neu aufbauen können! War sein einziger Ausweg aus dem Dilemma die Vortäuschung eines Suizidversuchs, um Rettung und Aufmerksamkeit herbeizuführen? Mit dem Anruf bei der Notrufzentrale konnte er dafür sorgen, dass Rettung kommen würde, und dann das Schicksal entscheiden lassen. So konnte er den Mittelweg zwischen den beiden Alternativen, zu leben oder zu sterben, gehen, sich von allem befreien und die Kontrolle über diese Entscheidung in die Hand anderer legen. Ein geschickter, aber potenziell tödlicher Schachzug.

Nach einer langen Zeit auf der Intensivstation kann Herr Novak seine Rehabilitation beginnen und wird in ein Pflegeheim verlegt. Er befindet sich noch immer in psychiatrischer Betreuung, doch es zeigen sich Besserungen. Ich erfahre, dass seine Suizidgedanken seltener würden und dass er wieder Freude am Leben gewinne. Nur seine Kinder fehlten ihm, seine Frau verbiete noch immer den Kontakt. Im Nachhinein sei er aber dankbar, dass man ihn gerettet habe. Worte, die mich zuversichtlich stimmen. Auch wenn Krankengeschichten am Anfang komplex und verschachtelt wirken, kann alles ein gutes Ende nehmen. Denn auch wenn beim Eintreffen eines Patienten im Spital vieles für einen Therapieabbruch spricht, lohnt es sich, den Fall - und den Menschen dahinter - in allen Details anzuschauen und immer wieder interdisziplinär zu besprechen.

Im Fall Novak haben die Ärzte richtig entschieden. Doch ist das immer so? Ungewissheit gehört wohl zu diesem Beruf, und damit muss ich in Zukunft leben lernen. In diesem Sinne schaue ich meiner späteren Tätigkeit als Ärztin mit Interesse und Faszination, aber auch mit Respekt entgegen. 